\title{
Brownian dynamics simulations of ionic current through an open channel
}

\author{
R. Tindjong*, R.S. Eisenberg ${ }^{\dagger}$, I. Kaufman**, D. G. Luchinsky* and \\ P.V.E. McClintock* \\ *Department of Physics, Lancaster University, Lancaster LA1 4YB, UK. \\ 'Department of Molecular Biophysics and Physiology, Rush Medical college, \\ 1750 West Harrison, Chicago, IL 60612, USA. \\ ${ }^{* *}$ VNII for Metrological Service, Gosstandart, Moscow, 119361, Russia.
}

\begin{abstract}
Ionic motion through an open ion channel is analyzed within the framework of selfconsistent Brownian dynamics. A novel conceptual model in which the ions motion is coupled to the vibrations of the pore walls is introduced. The model allows to include into simulations an important additional mechanism of energy dissipation and the effects of self-induced strong modulation of the channel conductivity.
\end{abstract}

Keywords: ion channels, Poisson equation, Langevin equation, self-consistent approach PACS: 87.16.Uv

\section{INTRODUCTION}

Ion channels in the cell membrane are natural nanotubes that control a vast range of physiological activity [1]. Understanding their structure-function relationship is the main challenge. Much progress has been made in that direction in recent years. Theories ranging from Poisson Nernst Planck (PNP) theory , Brownian dynamics (BD) simulations and Molecular Dynamics (MD) have been developed, each of them presenting its own particular strengths and weaknesses [2]. In our model, we use a one dimensional approximation of the ion dynamics inside the channel. To this end, we neglect the off-axis motion of ions in the channel to be able to include in an effective way interaction with the channel wall. One of the goals of the simulations is to infer the diffusion coefficient for ionic motion in the channel, rather than assuming it to be known. To this end we therefore integrate out fast degrees of freedom related to the ion motion perpendicular to the channel axis. This effectively reduces the problem to that of one-dimensional motion along the reaction coordinate, which is in our case the channel axis. And the reaction is the transition of ion from the left to the right (or vice versa). This approach is standard in chemical kinetics, allowing self-consistent treatment of ionic diffusion in an open protein pore at the level of the Brownian motion approximation. Our approach allows us to introduce, in addition another very important degree of freedom related to the wall motion. The latter is a source of both self-induced enhancement of transition probabilities and a real source of dissipation. 


\section{MATERIALS AND METHODS}

We start our analysis by considering the Langevin equation for the $i$-th ion when the channel walls are fixed, which takes the form:

$$
m_{i} \dot{\mathbf{v}}_{i}=-m_{i} \gamma_{i} \mathbf{v}_{i}+\mathbf{f}_{i}+\sqrt{2 \gamma_{i} m_{i} k_{B} T} \xi_{i}(t)
$$

where $m_{i}$ and $\mathbf{v}_{i}$ are the mass and the velocity of the ion. The friction force is proportional to $\mathbf{v}_{i}$ with a coefficient of proportionality $m_{i} \gamma_{i}$. The equation for a $j$-th different type of ion is written in the same way, but with different mass and friction coefficient. The force acting upon the ion has a number of components

$$
\mathbf{f}_{i}=\mathbf{f}_{D}+\mathbf{f}_{E}+\mathbf{f}_{R}
$$

where $\mathbf{f}_{D}$ is the dielectric force due to the channel walls, fixed charge, and external applied potential, $\mathbf{f}_{E}$ is the electrostatic force due to the Coulomb interaction between moving ions, finally $\mathbf{f}_{R}$ is a short range repulsive force. We accommodate the short range repulsive potential through the following expression [3]

$$
U_{s r}(r)=\frac{F_{0}}{9} \frac{\left(r_{1}+r_{2}\right)^{10}}{r^{9}},
$$

where $r_{1}$ and $r_{2}$ are the Pauling radii of the ions, $r$ is the ion-ion distance, and $F_{0}$ is a short-range force constant taken to be $F_{0}=2 \times 10^{-10} \mathrm{~N}$.

Eqs. (3) can be integrated simultaneously in the bulk solution. A snapshot of ions near the channel mouth is shown in Fig.1
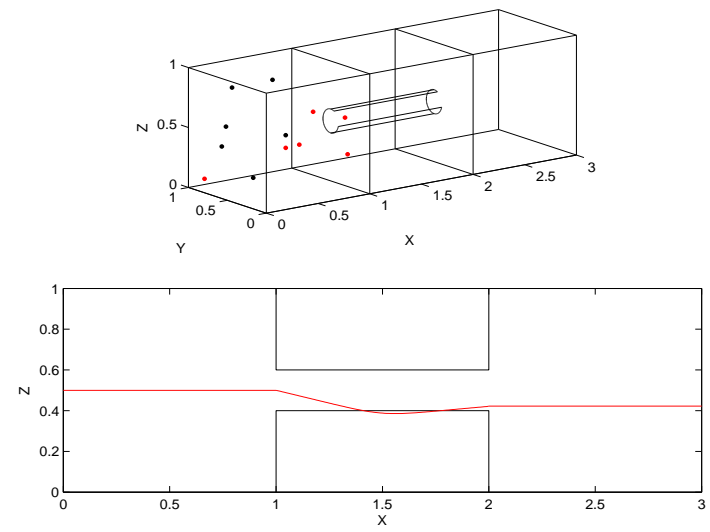

FIGURE 1. A snapshot of ions in the bulk solution obtained by simultaneous solution of Eqs. (1).

The line in the bottom figure is a representation of the one-dimensional approximation of the potential across the channel. The shift between the two ends is the potential drop due to the applied voltage. As soon as an ion enters the channel we assume that its motion is one dimensional along the axis of the channel. In particular this means that the multiple interactions of the ion with the wall during its diffusion within the channel 
are included in the unknown diffusion coefficient, which depends in turn on the position of the ion in the channel. We note that such an approximation is traditional in chemical physics where reaction in a system with many degrees of freedom is often considered as a one-dimensional dynamics along the so-called "reaction coordinate". In the present case, reaction is the transition of an ion from the left volume to the right (or vice versa), and the reaction coordinate is the $x$-coordinate along the channel axis.

For the ion in the channel the main driving force is $f_{D}$. Since we have assumed symmetry of the system about the channel axis, we calculate the corresponding force by integrating the Poisson equation using the Finite Volume Method in the system shown in the Fig.1, with only the ions within the channel taken into account.

In the bulk reservoir, we consider a symmetric concentrations of $\mathrm{NaCl}$. We used a stochastic boundary condition. Each time that an ion cross the channel, it is re-injected back into a random location with zero velocity. This way, we maintain fixed the specified concentrations in the reservoirs. The transmembrane potential which is generated by the applied electric potential across the membrane far away from the channel is explicitly taken into account in the solution of Poisson's equation.

\section{RESULTS AND DISCUSSION}

Before using the Poisson's equation solution, we make sure that the latter is sufficiently robust by comparing the numerical potential with a simple electrostatic problem. For example, the program reproduces accurately the potential created by one charge in a space divided in two with two different dielectric coefficients. After this check, we feel confident in using the numerical simulation of the Poisson equation to estimate the energy faced by an ion entering the channel. A $\mathrm{Na}^{+}$entering the channel is trapped in the energy well created by a negatively charged ring located at the middle of the channel, as can be seen on Fig.2

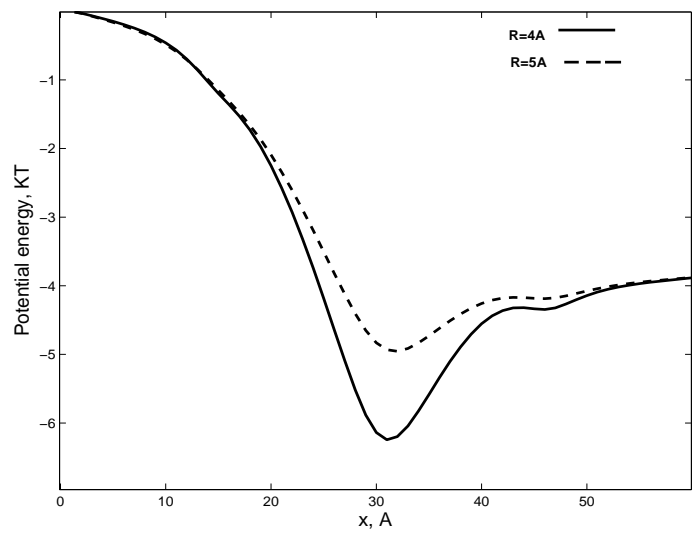

FIGURE 2. Profile of the Energy faced by a $N_{a}^{+}$ion.

For each applied potential, the Langevin equation is solved. The trajectories of ions traversing the channel are tracked. By counting all the transitions of ions crossing the 
channel during a given period of time, the current can be calculated for each applied voltage. The current-voltage characteristic of the channel can thus be obtained. This can then be compared with experiments. The position-dependent diffusion coefficient of the ion as it moves across the channel has also to be calculated. As can be seen in Fig.2, the variation of the channel radius due to its interaction with the ion moving in the channel has a huge effect on the potential energy. The ion will face a smaller barrier for larger channel radius. The equations of motion for the moving wall and for the single ion in the channel can be written as:

$$
\begin{aligned}
m_{i} \ddot{x}_{i} & =-m_{i} \gamma_{i} \dot{x}_{i}+\mathbf{f}_{i}+\frac{q_{1} q_{2}|(x-L / 2)|}{4 \pi \varepsilon \varepsilon_{0} \tilde{R}^{3}}+\sqrt{2 \gamma_{i} m_{i} k_{B} T} \xi_{i}(t), \\
M \delta \ddot{r}+M \tilde{\Gamma} \delta \dot{r}+k \delta \tilde{r} & =-\frac{q_{1} q_{2} \delta \tilde{r}}{4 \pi \varepsilon \varepsilon_{0} \tilde{R}^{3}}+\sqrt{2 M \tilde{\Gamma} k_{B} T} \tilde{\eta}(\tilde{t}),
\end{aligned}
$$

Here $\tilde{R}=\sqrt{(x-L / 2)^{2}+\delta \tilde{r}^{2}}$ is the distance between moving ion on the channel axis and charged vibrating segment of the wall, and $q_{1}$ and $q_{2}$ are respectively the charge of the moving ion inside the channel and the charged ring located at the middle of the channel.

\section{CONCLUDING REMARKS}

We have presented a self-consistent method for determination of the current-voltage characteristic of the ion channel. So far, we have successfully calculated the energy barrier faced by a $\mathrm{Na}^{+}$ion traversing the channel. Studies of the bi-ionic solution and the effect of the flexible protein wall, as well as the space dependant diffusion coefficient, are in progress. As results, we expect the coupling of ionic motion with vibration of the wall to introduce energy dissipation and self-induced modulation of the transition probability through the ion channel.

\section{ACKNOWLEDGMENTS}

The work was supported by the Engineering and Physical Sciences Research Council (UK), The Russian Foundation for Basic Research, INTAS, and ESF.

\section{REFERENCES}

1. B. Hille, Ionic Channel Of Excitable Membranes, Sinauer Associates, Sunderland, MA, 1992.

2. Gennady V. Miloshesvky and Peter C. Jordan, "Permeation in ion channels: the interplay of structure and theory" TRENDS in Meurosciences 27 (6),pp. 308-314, 2004

3. G. Moy, B. Corry, S. Kuyucak, and S.-H. Chung, "Tests of continuum theories as models of ion channels. I. Poisson-Boltzmann theory versus Brownian dynamics," Biophys. J. 78(5), pp. 2349-2363, 2000 . 
Copyright of AIP Conference Proceedings is the property of American Institute of Physics. The copyright in an individual article may be maintained by the author in certain cases. Content may not be copied or emailed to multiple sites or posted to a listserv without the copyright holder's express written permission. However, users may print, download, or email articles for individual use. 